\title{
PERSUASIVE TECHNOLOGY TO GENERATE SONGS PLAYLIST USING EMOTION BASED MUSIC PLAYER
}

\author{
Ms. Uma Yadav \\ Assistant Professor \\ Department of CSE, \\ Member COE Digital Technology \\ G H Raisoni College of Engineering, \\ Nagpur, Maharashtra, India
}

\begin{abstract}
The advanced approach that offers the user with automated generated playlist of songs based on the mood of the user. In today's world everyone uses the music to relax him or herself. To automate the Playlist generation process lots of algorithm were developed and proposed. Emotion Based Music Player aims at perusing and inferring the data from facial expressions and creating a Playlist based on the parameters extracted. Human moods are proposed for common understanding and sharing feelings and aims. Depending upon the current mood of the user the player automatically selects the song and plays it. The proposed system focuses on developing the Emotion Based Musing Player by detecting the human emotions through facial expression extraction technique. The proposed system works on Playlist generation and Classification of Emotions. The system is designed in such a way that the Facial expressions are captured through an inbuilt camera, analyze the extracted features of the image and determines the mood of the user and arranges the playlist accordingly.
\end{abstract}

Keywords- Persuasive Technology, Face Detection, Feature Extraction, Emotion Classification, Mood Detection, Music Playlist Generation, Audio-Feature, CNN Approach

\section{INTRODUCTION}

To enhance the individual's life Music plays a very important role and it is the medium of entertainment for Music lovers and listeners. In the Todays Era with the technology advancement in the field of Multimedia, multiple music players have been developed with various options like Fast forward, Reverse, local playback, mutable playback speed (seek \& time compression), streaming playback with multicast streams and including volume variation, genre classification etc. Even though these options satisfy the basic requirement of user, the user has to tackle the task of manually browsing the

\author{
Ms. Shweta Kharat \\ Assistant Professor \\ Department of CSE, \\ Member COE Digital Technology \\ G H Raisoni College of Engineering, \\ Nagpur, Maharashtra, India
}

songs playlist and select songs based on his current temper and actions. The proposed novel approach is different from traditional approach as it reduces the task of manual browsing the songs playlist. To stop labor emotion marking work we suggested in our work, a generic recommendation model for emotion-based music to recommend your music. The proposed system uses the Persuasive effective Technique [10][14] that would generate a songs playlist based on present mood and activities of the user. The most natural way of expressing the feeling is facial expression by means of which we can detect the present mood and behaviour, and for this the proposed algorithm requires less computation, time, and cost. In particular, we examined the extraction and modification of music features, the algorithm for discovering the relationship between musical characteristics and feelings has been proposed. The facial expressions are broadly categorized into 5 such as Anger, Joy, Surprise, Sad and Excitement. The proposed emotion model classify a song based on 7 classes of emotions i.e. anger, joy, sad, joy-anger, joy-surprise, sadanger, joy-excitement.

Every day, most individuals encounter music with affective responses. Joy, for example, while listening to an exceptional performance at the concert, the sorrow of listening to late night movie songs. Some scholars have been committed to understand the interactions between music and metaphysical, musicological feelings, anthropological and psychological views. Another potential solution is to learn the rules by

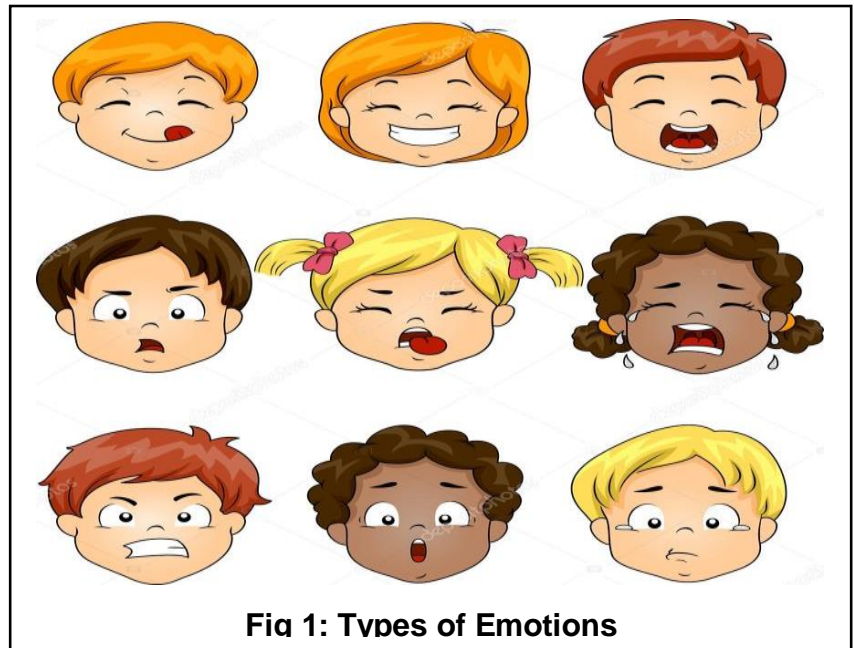




\section{International Journal of Engineering Applied Sciences and Technology, 2021 \\ Vol. 5, Issue 10, ISSN No. 2455-2143, Pages 119-123 \\ Published Online February 2021 in IJEAST (http://www.ijeast.com)}

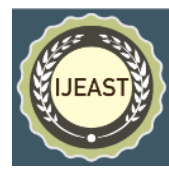

training with emotion forms labeled from music. This approach is however, time consuming.

\section{LITERATURE REVIEW}

To classify human behaviour and emotional state lots of technique and approaches were proposed and developed.

The Author Anagha S. Dhavalikar et al [1] proposed the automatic recognition of facial expression. The author has built the recognition system to detect the face and facial expression. Basically the author has involved three phases such as a) Face Detection b) Feature Extraction and c) Expression recognition. In the face detection phase the author has used the RGB Color modelling, to get the face the author has used lighting compensation and to retain the face such as eyes and mouth the author has used morphological operations. For facial feature extraction they have used Active Appearance Model Method. Data files are created using located points on face such as eye, eyebrows and mouth and detect the face where expressions are given as an input, according to expression AAM model changes.

Author Yong-Hwan Lee et al [2] proposed the system based on Bezier curve fitting. Two steps were used for facial expression and emotion detection by proposed system. In First step from the original input image the detection and analysis of face area takes place and in second step the verification of facial emotion characteristics feature in the ROI takes place. For face detection the first phase uses the color image based on skin color by initializing spatial filtering, based on lighting compassion result, it estimates the face position and location of eye and mouth which is used for feature map. After the region of interest extraction the system cuts the points of feature map by applying Bezier Curve on eye and mouth. For emotion understanding the proposed system uses training and calculating the difference of Hausdorff distance between entered face image and image from database using Bezier curve.

Arto Lehtiniemi et al [3] for music recommendation the author has proposed animated mood picture. Here the user relate with the group of images to obtain music recommendation similar to the picture. Here the user interacts with a set of images in the implemented prototype to receive new music suggestions from related genres. This shows that photographs of mood are a promising way of accessing music collections and discovering new music. Nokia research centre has developed the mentioned music recommendation system. To describe the genre and Audio Signal Processing they have used Meta tags.

F. Abdat et al [4] proposed fully automatic facial expression and recognition system. The author has performed operation on three phases such as detection of Face, Extraction of Facial Characteristics and classification of Facial expression. To detect faced feature point the proposed system used Anthropometric Model. The method involves the variation of 21 distances which basically describes neural face features and for the classification they have used SVM. Experimental findings show that the proposed solution is a successful way to identify emotions with an emotion recognition rate of more than 90 percent in real time via facial expression. Based on the change in the emotional state of the computer user, this method is used to monitor the music player.

Fang-Fei Kuo et al [5] proposed novel approach for emotion based music system which involves film music association discovery. The author has investigated the extraction of music features and reformed the affinity graph for interaction exploration between emotions and music features. In order to construct the model of music recommendation, the author suggested the phase including the extraction of the music features from film Music, the identification of feelings from the film section, and the exploration of the relations between musical characteristics and emotions. Based on the query emotions, with respect to the query, the recommendation model will return the recommended music features. The suggested features are then used to rate the music from the database and to recommend music for query feelings. In their experimental results they have shown that they have achieved $85 \%$ accuracy in average.

Ivana Andjel kovic et al [6] proposed MoodPlay a Hybrid approach for Music recommendation system which incorporates content and mood-based filtering in a collaborating boundary The author explained how MoodPlay helps the user to explore a set of music across latent affective dimensions, and also explained how to combine user feedback with predictions based on a pre-existing user profile at reference time. A user research $(\mathrm{N}=240)$ was addressed by the author, with four conditions being evaluated with different degrees of visualization, interaction and control. Results show that in a latent room, visualization and interaction enhance acceptance and comprehension of both metadata and recommendations for objects. Too much of either, however can lead to cognitive overload and a negative effect on user experience.

Anukriti Dureha et al [7] proposed an Accurate Algorithm for Engendering a Music Playlist based on Facial Expressions. According to current emotional state of a user the author concluded that manual separation of the playlist and annotation of songs is time consuming process. According to him many algorithms were proposed to automate this process but still the existing algorithm are quite slow and expensive. The author proposed an algorithm based on a user's facial expressions, which automates the process of creating an audio playlist. The proposed algorithm 


\section{International Journal of Engineering Applied Sciences and Technology, 2021 \\ Vol. 5, Issue 10, ISSN No. 2455-2143, Pages 119-123 \\ Published Online February 2021 in IJEAST (http://www.ijeast.com)}

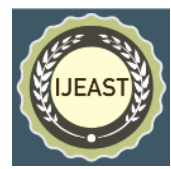

reduces the designed system's total computing time and cost. It also focuses on the cumulative accuracy of the device being designed.

Bruce Ferwerda et al [8] Proposed Enhanced Music Recommender System by using Personality Information and Emotional States. The author proposed that by including personality and emotional states the initial research assumptions can improve music recommendations. The accuracy of the recommendation system can be enhanced by considering psychological factors. Basically, the framework suggested devotes itself to how people use music to change their emotional states and how their personality is connected to these directives. The author used Twitter feeds in his analysis to extract personality and emotional states, from which he initially trusted various existing methods and combined them to boost parameter predictability. With the results of the above steps, the algorithm will start matching music that matches the emotion control method of the consumer. The author initially relies on the emotional tags provided by Last FM to find suitable music.

H. Immanuel James et al [9] proposed the system which emphasis on detecting human emotions for evolving emotion based music player. Here the author has used pycharm tool for analysis. For faster faced detection operation and to reduce the computational time the author has used the linear classifier instead of the SVM. In Methodology the author has used SVM [11][15] for image pre-processing, image segmentation, image feature extraction and classification.

\section{Proposed Methodology}

In improving the life of the person, music plays a very important role, as it is an important source of entertainment for music lovers and listeners. A number of music players have been developed in today's world [12], with everincreasing advances in the field of multimedia and technology, with features such as Fast forward, Reverse, local playback, mutable playback speed (seek \& time compression), streaming playback with multicast streams and including volume variation, genre classification etc.

Following modules included in the Proposed Methodology,

3.1 Emotion Capture Module: Image will be captured through phone's camera. Then it will go for the tone mapping to adjust the original contrast. After this the image will be converted to binary and the face is detected using the Viola-Jones Algorithm. Most studies have been performed on facial emotion recognition, Voice, text and physiological signals. In fact, there is Research that extracts feelings from the audio description given for people with visual impairments [13]. For each of the selected 22 types of emotion tokens, a list of emotion tokens was created in this work. The presence of emotional signs is a symptom of Emotion that is represented.

3.2 Audio-Feature Capture Module: The elements of music that influence emotion include melody, rhythm, Tempo, mode, key, harmony, tone-color and dynamics. Among these elements of song, melody, fashion, tempo and rhythm have stronger effects on feelings. In general, the major is brighter, happier than minor; more thrilling or exciting is the fast tempo. It's more nervous than a sluggish tempo. In this, the input will be songs list. They have to undergo the pre-processing to clean them up. The preprocessed data will be used for Feature Extraction. Features like rhythm, pitch, toning etc. will be used to categorize each song.

3.3 Emotion-Audio Matching Module: Emotions Captured from the camera is stored in database and will be used to match the categories of songs. The emotion module and audio module are finally mapped and combined using an Emotion-Audio Matching Module.

3.4 Upload Module: Songs can be uploaded category-wise and can be played in the music player.

\section{ARCHITECTURAL FLOW}

The Fig.2 flowchart describes overall working of The Emo. When you start the app first time after installation, it shows a nice short dialogue box that we don't store any data from your device. Clicking Continue button there will lead you to our homepage which is separated into two parts. The upper part has a big yellow camera image (gif actually) which is for taking pictures of course, but what it does is, it takes your picture and performs all the operations shown in the upper part of flowchart (i.e. our ML algorithm) and when it's done. It returns the output as a string representing your mood. Based on the output the system recommends the songs playlist.

So, in simple words, the first part of the homepage is for automatically guessing your mood and providing you with the songs that you can listen when you're feeling that one. The second part is for manually selecting your mood in case you have some songs in your mind, We provided the buttons for the moods that we have in store, clicking those will lead you to list of songs of the corresponding mood. The next Screen is for upload options. In case we don't have songs of your choice, we provided you with this option, it's located at the upper-right corner of the screen in the App bar, clicking it will take you to a dialogue box where you will be given options for different moods selecting any one will upload your songs in that class (or category) the next time you're in that mood you will see your added songs are in the list and you can listen to them. not 
only the user every Emo user will get those songs and lastly, I want to describe our algorithm in brief same as the flowchart does. So, our method starts with taking the image as input, and then we perform face detection, it basically removes the parts of images that are not relevant in other words, it captures the face only. Then we detect the faces in the picture, this step returns the number of faces detected (also only human face counts), all faces other than human is discarded. Next step is to extract the facial landmarks from the detected faces such as nose, eyes, lips etc. These we get in the form of their $(x, y)$ co-

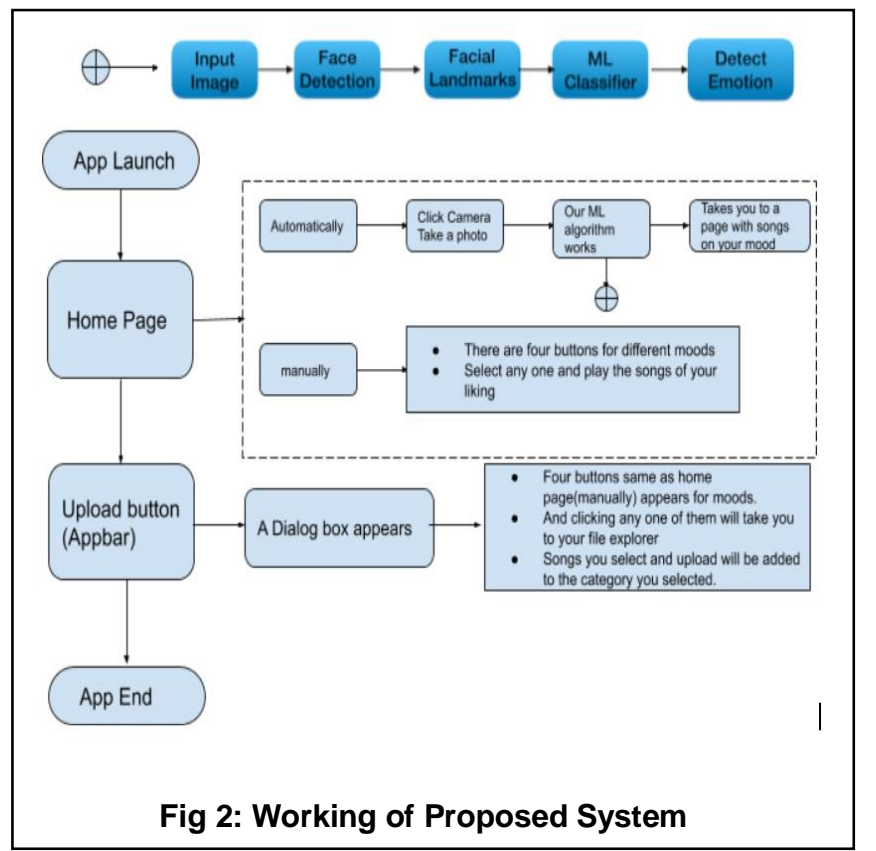

ordinates on the image. Then based on some factors we have trained a classifier, it takes those factors of the input image and assigns them an importance level, and then based on those we get our Emotion from the image.

\section{RESULTS AND IMPLEMENTATION}

5.1 Face Detection: In first step, the proposed method starts with taking the image as input, and then it performs face detection, it basically removes the parts of images that are not relevant in other words, it captures the face only. Then we detect the faces in the picture, this step returns the number of

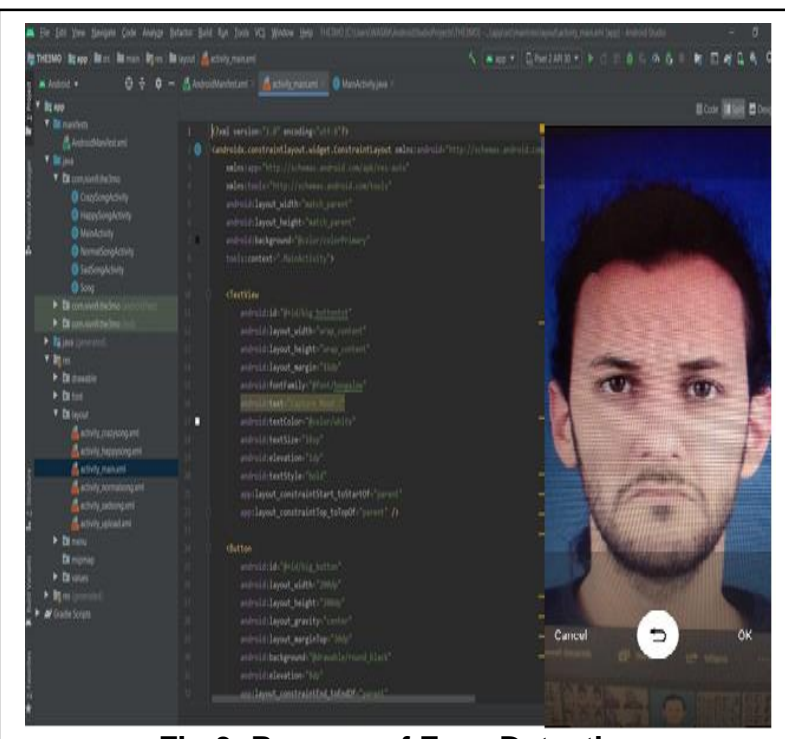

Fig 3: Process of Face Detection faces detected (also only human face counts), all faces other than human is discarded.

\subsection{Facial Expression extraction:}

In Next step it extracts the facial landmarks from the detected faces such as nose, eyes, lips etc. These we get in the form of their (x, y) co-ordinates on the image. Then based on some factors we have trained a classifier, it takes those factors of the input image and assigns them an importance level, and then based on those we get our Emotion from the image.

5.3 Music Playlist Generation: In last step based on emotion classified it generates the playlist of songs based on the results of the algorithm.

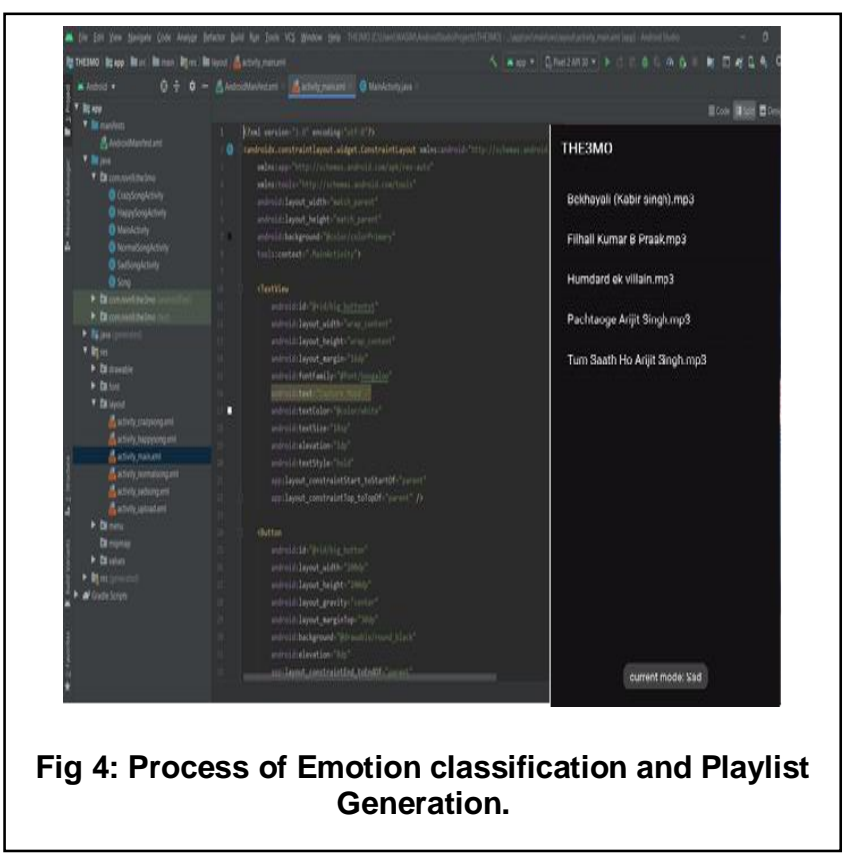

The results of proposed system are shown below, in fig 3 first the face of the human is detected and also classify the number of faces detected.

\section{CONCLUSION}

This paper introduces a music playlist, a modern way of using machine learning methods to personalize song playlists. Our solution works and provides preferable playlists for better users. The performance of the CNN method is higher than the Random Forest Classifier algorithms because of the large number of classes. Since the experiment was carried out on a small dataset and a limited number of features, by incorporating more features such as age, weather, etc., it can still be enhanced. More attributes would make decision-making and song prediction improvable. What user has his or her own preferences on what kind of song for the appropriate mood should be played. For example some people, when they are 


\section{International Journal of Engineering Applied Sciences and Technology, 2021 \\ Vol. 5, Issue 10, ISSN No. 2455-2143, Pages 119-123 \\ Published Online February 2021 in IJEAST (http://www.ijeast.com)}

sad, listen to sad songs, while some may choose happy songs to improve their mood. Collecting this information from every user will help us create a better radio application unique to the user. The implementation of this prototype in current music applications will provide users with a better music experience.

\section{REFERENCES}

[1] Dhavalikar, A. S., \& Kulkarni, R. K. (2014). Face detection and facial expression recognition system. 2014 International Conference on Electronics and Communication Systems, ICECS 2014. https://doi.org/10.1109/ECS.2014.6892834

[2] Lee, Y. H., Han, W., \& Kim, Y. (2013). Emotional recognition from facial expression analysis using bezier curve fitting. Proceedings - 16th International Conference on Network-Based Information Systems, NBiS 2013. https://doi.org/10.1109/NBiS.2013.39

[3] Lehtiniemi, A., \& Holm, J. (2012). Using animated mood pictures in music recommendation. Proceedings of the International Conference on Information Visualisation. https://doi.org/10.1109/IV.2012.34

[4] Abdat, F., Maaoui, C., \& Pruski, A. (2011). Humancomputer interaction using emotion recognition from facial expression. Proceedings - UKSim 5th European Modelling Symposium on Computer Modelling and Simulation, EMS 2011.

https://doi.org/10.1109/EMS.2011.20

[5] Wang, T. H., \& James Lien, J. J. (2009). Facial expression recognition system based on rigid and nonrigid motion separation and $3 \mathrm{D}$ pose estimation. Pattern Recognition, 42(5). https://doi.org/10.1016/j.patcog.2008.09.035

[6] Andjelkovic, I., Parra, D., \& O’Donovan, J. (2019). Moodplay: Interactive music recommendation based on Artists' mood similarity. International Journal of Human Computer Studies, 121. https://doi.org/10.1016/j.ijhcs.2018.04.004

[7] Dureha, A. (2014). An Accurate Algorithm for Generating a Music Playlist based on Facial Expressions. International Journal of Computer Applications, 100(9). https://doi.org/10.5120/17557-8163

[8] Ferwerda, B., \& Schedl, M. (2014). Enhancing music recommender systems with personality information and emotional states: A proposal. CEUR Workshop Proceedings, 1181.

[9] James H. Immanuel, J. James Anto Arnold, J. Maria Masilla Ruban, Tamilarasan M., Saranya R.(2019),
"EMOTION BASED MUSIC RECOMMENDATION SYSTEM", International Research Journal of Engineering and Technology (IRJET), Volume 6,.

[10] Yadav, U. D., \& Mohod, P. S. (2013). Adding persuasive features in graphical password to increase the capacity of KBAM. 2013 IEEE International Conference on Emerging Trends in Computing, Communication and Nanotechnology, ICE-CCN 2013. https://doi.org/10.1109/ICE-CCN.2013.6528553

[11] Khedikar, S. V., \& Yadav, U. (2018). Detection of disease from radiology. Proceedings of 2017 International Conference on Innovations in Information, Embedded and Communication Systems, ICIIECS 2017, 2018-January. https://doi.org/10.1109/ICIIECS.2017.8276174

[12] Kabani Hafeez, Khan Sharik, Khan Omar, Tadvi Shabana(2015). Emotion Based MusicPlayer. International Journal Engineering Research and General Science Volume 3, Issue 1, January-February.

[13] Yadav, U., Bondre, S., Maidamwar, P., \& Khobragade, P. (2019). AN AIANDML BASED APPLICATION FOR VISUALLY IMPAIRED PEOPLE. International Journal of Engineering Applied Sciences and Technology, 04(07). https://doi.org/10.33564/ijeast.2019.v04i07.038

[14] Yadav U, Sakure S (2015). Graphical Password Knowledge Based Authentication System Enhancement using Persuasive Technology. International Journal of Science and Research (IJSR), 04(11).

[15] Khedikar S, Yadav U. (2017). Identification of Disease by Using SVM Classifier. International Journal of Advanced Research in Computer Science and Software Engineering, 07(04). 